\title{
Erratum
}

\section{Erratum to: On the perturbative renormalization of four-quark operators for new physics}

\author{
M. Papinutto ${ }^{1}$, C. Pena ${ }^{2,3}$, D. Preti ${ }^{3, \text { a }}$ \\ ${ }^{1}$ Dipartimento di Fisica, "Sapienza" Università di Roma, and INFN, Sezione di Roma, Piazzale A. Moro 2, 00185 Rome, Italy \\ 2 Departamento de Física Teórica, Universidad Autónoma de Madrid, Cantoblanco, 28049 Madrid, Spain \\ ${ }^{3}$ Instituto de Física Teórica UAM-CSIC, Universidad Autónoma de Madrid, c/Nicolás Cabrera 13-15, Cantoblanco, 28049 Madrid, Spain
}

Published online: 12 January 2018

(C) The Author(s) 2018. This article is an open access publication

Erratum to: Eur. Phys. J. C (2017) 77(6):376 https://doi.org/10.1140/epjc/s10052-017-4930-6

This erratum concerns the correction of some typographic errors appearing in the second and third sections of the article. The errors do not affect any of the results presented.

- The definitions of operators $Q_{5}$ and $\mathcal{Q}_{5}$ in Eq. (2.1) should read

$$
Q_{5}^{ \pm}=-2 \mathcal{O}_{\mathrm{TT}}^{ \pm}, \quad \mathcal{Q}_{5}^{ \pm}=-2 \mathcal{O}_{\mathrm{T} \tilde{\mathrm{T}}}^{ \pm} .
$$

- To simplify the notation, from Eq. (2.7) on, the superscript \pm is omitted.

- In Eqs. (2.8), (2.9), (2.18), (2.21), (2.26) and (2.36) the partial derivative should be replaced with a total derivative.
- In Eq. (2.16) the - sign should be replaced with a + sign.

- In Eq. (3.15) $G_{k ; s}^{ \pm}\left(x_{0}\right)$ should be replaced with $G_{k ; s}^{ \pm}(T-$ $\left.x_{0}\right)$.

- From Eq. (3.25) until the end of Section 3, $Z$ should be replaced with $\mathcal{Z}$.

- Equation (3.25) should $\operatorname{read} \mathcal{Z}_{1 ; s, \alpha}^{ \pm} \mathcal{A}_{1 ; s, \alpha}^{ \pm}=\left.\mathcal{A}_{1 ; s, \alpha}^{ \pm}\right|_{g_{0}^{2}=0}$.

We thank A. Vladikas for his careful reading of this manuscript.

Open Access This article is distributed under the terms of the Creative Commons Attribution 4.0 International License (http://creativecomm ons.org/licenses/by/4.0/), which permits unrestricted use, distribution, and reproduction in any medium, provided you give appropriate credit to the original author(s) and the source, provide a link to the Creative Commons license, and indicate if changes were made. Funded by SCOAP ${ }^{3}$.

The original article can be found online at https://doi.org/10.1140/ epjc/s10052-017-4930-6.

a e-mail: david.preti@csic.es 